\title{
On Polynomial Systems Arising from a Weil Descent
}

\author{
Christophe Petit* and Jean-Jacques Quisquater ${ }^{\star \star}$ \\ UCL Crypto Group, \\ Université catholique de Louvain \\ Place du Levant 3 \\ 1348 Louvain-la-Neuve (Belgium) \\ $\{$ christophe.petit,jjq\}@uclouvain.be
}

\begin{abstract}
In the last two decades, many computational problems arising in cryptography have been successfully reduced to various systems of polynomial equations. In this paper, we revisit a class of polynomial systems introduced by Faugère, Perret, Petit and Renault. Based on new experimental results and heuristic evidence, we conjecture that their degrees of regularity are only slightly larger than the original degrees of the equations, resulting in a very low complexity compared to generic systems. We then revisit the application of these systems to the elliptic curve discrete logarithm problem (ECDLP) for binary curves. Our heuristic analysis suggests that an index calculus variant due to Diem requires a subexponential number of bit operations $O\left(2^{c n^{2 / 3} \log n}\right)$ over the binary field $\mathbb{F}_{2^{n}}$, where $c$ is a constant smaller than 2. According to our estimations, generic discrete logarithm methods are outperformed for any $n>N$ where $N \approx 2000$, but elliptic curves of currently recommended key sizes $(n \approx 160)$ are not immediately threatened. The analysis can be easily generalized to other extension fields.
\end{abstract}

\section{Introduction}

While linear systems of equations can be efficiently solved with Gaussian elimination, polynomial systems are much harder to solve in general. After their introduction by Buchberger [13], Gröbner bases have become the most popular way to solve polynomial systems of equations, in particular since the development of fast algorithms like $\mathrm{F}_{4}$ 26] and $\mathrm{F}_{5}$ [27. Polynomial systems arising in cryptography tend to have a special structure that simplifies their resolution. In the last twenty years, many cryptographic challenges have been first reduced to polynomial systems of equations and then solved with fast and sometimes dedicated Gröbner basis algorithms [42,30 38|10|22 23|32|12|31].

\footnotetext{
* Supported by an F.R.S.-FNRS postdoctoral research fellowship at Université catholique de Louvain, Louvain-la-Neuve.

** This work was partly supported by the Belgian State's IAP program P6/26 BCRYPT.
} 


\section{Our Contribution}

In this paper, we revisit a particular class of polynomial systems introduced by Faugère et al. 3334. These systems naturally arise by deploying a multivariate polynomial equation over an extension field into a system of polynomial equations over the ground prime field (a technique commonly called Weil descent).

We first observe that polynomial systems arising from a Weil descent are a natural generalization of a well-known family of polynomial systems appearing in the cryptanalysis of HFE 48,42 18 30 38 24 10 22 23. Starting from this observation, we extend various experimental and theoretical results on HFE to the more general class of polynomial systems arising from a Weil descent. Our results suggest that the degrees of regularity of these systems are only sligthly larger than the degrees of their equations, essentially as small as they could be.

Following [34, we subsequently study an elliptic curve discrete logarithm algorithm of Diem [21] in the case of binary fields. Based on our heuristic analysis of polynomial systems arising from a Weil descent, we conjecture that the elliptic curve discrete logarithm problem can be solved over the binary field $\mathbb{F}_{2^{n}}$ in subexponential time $O\left(2^{c n^{2 / 3} \log n}\right)$, where $c$ is a constant smaller than 2 . For $n$ prime, this problem was previously thought to have complexity $O\left(2^{n / 2}\right)$.

Our analysis of polynomial systems arising from a Weil descent can also be applied to the factorization problem in $S L\left(2, \mathbb{F}_{2^{n}}\right)$, to HFE and to other discrete logarithm problems. These applications will be discussed in an extended version of this paper [49. Although we focus on characteristic 2 in this paper, most of our results can be easily extended to other characteristics.

\section{Outline}

The remaining of this paper is organized as follows. Section 2 contains most of the notations and definitions used in the paper. Section 3 provides general background on algebraic cryptanalysis with Gröbner bases. Section 4 contains our new analysis of polynomial systems arising from a Weil descent. The application to Diem's algorithm is detailed in Section 5 and Section 6 concludes the paper.

\section{Definitions and Notations}

We mostly follow the notations introduced in 33. For any "small" prime $p$ and any $n \in \mathbb{Z}$, we write $\mathbb{F}_{p^{n}}$ for the finite field with $p^{n}$ elements. We see the field $\mathbb{F}_{p^{n}}$ as an $n$-dimensional vector space over $\mathbb{F}_{p}$ and we let $\left\{\theta_{1}, \ldots, \theta_{n}\right\}$ be a basis for $\mathbb{F}_{p^{n}} / \mathbb{F}_{p}$. With some abuse of notations, we use bold letters for all elements, variables and polynomials over $\mathbb{F}_{p^{n}}$ and normal letters for all elements, variables and polynomials over $\mathbb{F}_{p}$. If $x_{1}, \ldots, x_{N}$ are variables defined over a field $\mathbb{K}$, we write $R:=\mathbb{K}\left[x_{1}, \ldots, x_{N}\right]$ for the ring of polynomials in these variables. Given a set of polynomials $f_{1}, \ldots, f_{\ell} \in R$, the ideal $I\left(f_{1}, \ldots, f_{\ell}\right) \subset R$ is the set of polynomials $\sum_{i=1}^{\ell} g_{i} f_{i}$, where, $g_{1}, \ldots, g_{\ell} \in R$. We write $\operatorname{Res}_{x_{i}}\left(f_{1}, f_{2}\right)$ for the resultant of $f_{1}, f_{2} \in R$ with respect to the variable $x_{i}$. A monomial of $R$ is a 
power product $\prod_{i=1}^{k} x_{i}^{e_{i}}$ where $e_{i} \in \mathbb{N}$. A monomial ordering for $R$ is an ordering $>$ such that $m_{1}>m_{2} \Rightarrow m_{1} m_{3}>m_{2} m_{3}$ for any monomials $m_{1}, m_{2}, m_{3}$ and $m>1$ for any monomial $m$. The leading monomial $L M(f)$ of a polynomial $f \in R$ for a given ordering is equal to its largest monomial according to the ordering. Its leading term is the corresponding term. For any polynomial $f \in R$, we denote the set of monomials of $f$ by $\operatorname{Mon}(f)$. We measure the memory and time complexities of algorithms by respectively the number of bits and bit operations required. Actual experimental results are given in megabytes and seconds. We write $O$ for the "big O" notation: given two functions $f$ and $g$ of $n$, we say that $f=O(g)$ if there exist $N, c \in \mathbb{Z}^{+}$such that $n>N \Rightarrow f(n) \leq c g(n)$. Similarly, we write $o$ for the "small o" notation: given two functions $f$ and $g$ of $n$, we say that $f=o(g)$ if for any $\epsilon>0$, there exists $N \in \mathbb{Z}$ such that for any $n>N$, we have $|f(n)| \leq \epsilon|g(n)|$. Finally, we write $\omega$ for the linear algebra constant. Depending on the algorithm used for linear algebra, we have $2.376 \leq \omega \leq 3$.

\section{Background on Polynomial System Resolution}

Let $R$ be a polynomial ring and let $>$ be a fixed monomial ordering for this ring. A Gröbner basis [1319] of an ideal $I\left(f_{1}, \ldots f_{\ell}\right) \subset R$ is a basis $\left\{f_{1}^{\prime}, \ldots, f_{\ell^{\prime}}^{\prime}\right\}$ of this ideal such that for any $f \in I\left(f_{1}, \ldots f_{\ell}\right)$, there exists $i \in\left\{1, \ldots, \ell^{\prime}\right\}$ such that $\mathrm{LT}\left(f_{i}^{\prime}\right) \mid \mathrm{LT}(f)$. The first Gröbner basis algorithm was provided by Buchberger in his $\mathrm{PhD}$ thesis [13]. Lazard [4] later observed that computing a Gröbner basis is essentially equivalent to performing linear algebra on Macaulay matrices at a certain degree.

Definition 1 (Macaulay Matrix 45,46]). Let $R$ be a polynomial ring over a field $K$ and let $\mathcal{B}_{d}:=\left\{m_{1}>m_{2}>\cdots\right\}$ be the sorted set of all monomials of degree $\leq d$ for a fixed monomial ordering. Let $F:=\left\{f_{1}, \ldots, f_{\ell}\right\} \subset R$ be a set of polynomials of degrees $\leq d$. For any $f_{i} \in F$ and $t_{j} \in \mathcal{B}_{d}$ such that $\operatorname{deg}\left(f_{i}\right)+\operatorname{deg}\left(t_{j}\right) \leq d$, let $g_{i, j}:=t_{j} f_{i}$ and let $c_{i, j}^{k} \in K$ be such that $g_{i, j}=$ $\sum_{m_{k} \in \mathcal{B}} c_{i, j}^{k} m_{k}$. The Macaulay matrix $\mathcal{M}_{d}(F)$ of degree $d$ is a matrix containing all the coefficients $c_{i, j}^{k}$, such that each row corresponds to one polynomial $g_{i, j}$ and each column to one monomial $m_{k} \in \mathcal{B}_{d}$.

The idea behind Lazard's observation is linearization: new equations for the ideal are constructed by algebraic combinations of the original equations, every monomial term appearing in the new equations is treated as an independent new variable, and the system is solved with linear algebra. Gröbner basis algorithms like $\mathrm{F}_{4}$ [26] and $\mathrm{F}_{5}$ [27] successively construct Macaulay matrices of increasing sizes and remove linear dependencies in the rows until a Gröbner basis is found. Moreover, they optimize the computation by avoiding monomials $t_{j}$ that would produce trivial linear combinations such as $f_{1} f_{2}-f_{2} f_{1}=0$. The complexity of this strategy is determined by the cost of linear algebra on the largest Macaulay matrix occuring in the computation.

The degree of the largest Macaulay matrix appearing in a Gröbner basis computation with the algorithm F5 is called the degree of regularity $D_{\text {reg. }}$. For 
a "generic" sequence of polynomials $f_{1}, \ldots, f_{\ell} \in R$ (with $\ell \leq n$ ), this degree is equal to $1+\sum_{i=1}^{\ell}\left(\operatorname{deg}\left(f_{i}\right)-1\right)$ [6]. The degree of regularity can be precisely estimated in the case of regular and semi-regular sequences [6]8] and (assuming a variant of Fröberg conjecture) in a few other cases [2811. However, precisely estimating this value for other classes of systems (in particular for the various structured systems appearing in cryptanalysis problems) may be a very difficult task. In practice, the degree of regularity may often be approximated by the first degree at which a non trivial degree fall occurs during a Gröbner basis computation.

Definition 2. Let $R$ be a polynomial ring over a field $K$ and let $F:=\left\{f_{1}, \ldots, f_{\ell}\right\}$ $\subset R$. The first fall degree of $F$ is the smallest degree $D_{\text {firstfall }}$ such that there exist polynomials $g_{i} \in R$ with $\max _{i}\left(\operatorname{deg}\left(f_{i}\right)+\operatorname{deg}\left(g_{i}\right)\right)=D_{\text {firstfall }}$, satisfying $\operatorname{deg}\left(\sum_{i=1}^{\ell} g_{i} f_{i}\right)<D_{\text {firstfall }}$ but $\sum_{i=1}^{\ell} g_{i} f_{i} \neq 0$.

We have $D_{\text {reg }} \geq D_{\text {firstfall }}$. For many classes of polynomial systems, the two definitions lead to very close numbers. Although this is not true in general (counterexamples can be easily produced), it seems to be true for "random systems" and "most real-life systems of equations" [38, p. 350] including HFE and its variants $30 / 382422$ 2311. This can intuitively be explained by the observation that an extremely large number of relations with a degree fall occur at the degree $D_{\text {firstfall }}$ or the degree $D_{\text {firstfall }}+1$ in these contexts, and these low degree relations can in turn be combined to produce lower degree relations [24, p. 561], until a Gröbner basis is finally found. In fact, the first fall degree has even sometimes been called degree of regularity in the cryptography community [24 22 23].

Many polynomial systems arising in cryptanalysis are very far from generic ones. In fact, their special structures often induce lower degrees of regularity, hence much better time complexities. Gröbner basis techniques have successfully attacked many cryptosystems, including $\mathrm{HFE}$ and its variants 48423038102223 , the Isomorphism of Polynomials 3212] and some McEliece variants 31. In many cases, the resolution of these systems could be accelerated using dedicated Gröbner basis algorithms that exploited the particular structures. As was first pointed out in 3334, this is also the case for polynomial systems arising from a Weil descent.

\section{Polynomial Systems Arising from a Weil Descent}

Let $n, n^{\prime}, m$ be positive integers and let $V$ be a vector subspace of $\mathbb{F}_{2^{n}} / \mathbb{F}_{2}$ with dimension $n^{\prime}$. Let $\mathbf{f} \in \mathbb{F}_{2^{n}}\left[\mathbf{x}_{\mathbf{1}}, \ldots, \mathbf{x}_{\mathbf{m}}\right]$ be a multivariate polynomial with degrees bounded by $2^{t}-1$ with respect to all variables. In [3334, Faugère et al. considered the following problem:

$$
\text { Find } \mathbf{x}_{\mathbf{i}} \in V, i=1, \ldots, m \text {, such that } \mathbf{f}\left(\mathbf{x}_{\mathbf{1}}, \ldots, \mathbf{x}_{\mathbf{m}}\right)=\mathbf{0} \text {. }
$$

The constraints $\mathbf{x}_{\mathbf{i}} \in V, i=1, \ldots, m$ are called linear constraints. From now on, we assume that $m n^{\prime} \approx n$ such that Problem (1) has about one solution on 
average. We also assume $n^{\prime} \geq t$. The multilinear case $(t=1)$ was first considered in 33] and later extended in 34.

Following 3334, Problem (1) can be reduced to a system of polynomial equations. Let $\left\{\theta_{1}, \ldots, \theta_{n}\right\}$ be a basis of $\mathbb{F}_{2^{n}}$ over $\mathbb{F}_{2}$ and let $\left\{\mathbf{v}_{\mathbf{i}} \mid i=1, \ldots, n^{\prime}\right\}$ be a basis of $V$ over $\mathbb{F}_{2}$. We define $m \cdot n^{\prime}$ variables $x_{i j}$ over $\mathbb{F}_{2}$ such that $\mathbf{x}_{\mathbf{i}}=\sum_{j=1}^{n^{\prime}} x_{i j} \mathbf{v}_{\mathbf{j}}$ and we group them into $m$ blocks of variables $X_{i}:=\left\{x_{i j} \mid j=1, \ldots, n^{\prime}\right\}$. By substituting each $\mathbf{x}_{\mathbf{i}}$ in $\mathbf{f}$, decomposing in the basis $\left\{\theta_{1}, \ldots, \theta_{n}\right\}$ and reducing by the field equations $x_{i j}^{2}-x_{i j}=0$, we obtain $\mathbf{0}=\mathbf{f}\left(\mathbf{x}_{\mathbf{1}}, \ldots, \mathbf{x}_{\mathbf{m}}\right)=$ $\mathbf{f}\left(\sum_{j=1}^{n^{\prime}} x_{1 j} \mathbf{v}_{\mathbf{j}}, \ldots, \sum_{j=1}^{n^{\prime}} x_{m j} \mathbf{v}_{\mathbf{j}}\right)=[\mathbf{f}]_{1}^{\downarrow} \theta_{1}+\ldots+[\mathbf{f}]_{n}^{\downarrow} \theta_{n}$ for some $[\mathbf{f}]_{1}^{\downarrow}, \ldots,[\mathbf{f}]_{n}^{\downarrow} \in$ $\mathbb{F}_{2}\left[x_{11}, \ldots, x_{m n^{\prime}}\right]$ that depend on $\mathbf{f}$ and on the vector subspace $V$. Problem (1) can therefore be reformulated as finding a solution to the (algebraic) system

$$
[\mathbf{f}]_{1}^{\downarrow}=0, \ldots,[\mathbf{f}]_{n}^{\downarrow}=0 .
$$

Due to the bounds on the degrees of $\mathbf{f}$, this system has a block structure: the degrees of all polynomials $[\mathbf{f}]_{k}^{\downarrow}$ are bounded by $t$ with respect to all blocks of variables. The resolution of System (2) can therefore be greatly accelerated using block-structured Gröbner basis algorithms [293334].

Link to HFE. In this paper, we observe that a particular instance of Problem (11) had previously been studied in the cryptography literature. Indeed, the well-known problem of inverting HFE [48|30|38] leads to a particular instance of System (2), where the polynomial $\mathbf{f}$ is univariate $(m=1)$ and the linear constraints are trivial $\left(V=\mathbb{F}_{2^{n}}\right)$ 1 Interestingly, although the polynomial $\mathbf{f}$ used in HFE has a particular shape (it leads to quadratic equations over $\mathbb{F}_{2}$ ), we will see that this shape has generically little influence on the complexity of Problem (1).

Ten years of research on HFE systems have shown that their degrees of regularity are abnormally low compared to generic systems, resulting in very efficient attacks. Although no definitive proof of these results has been published yet, the experimental observations of [30] are now being supported by theoretical evidence such as the isolation of a subsystem with less variables [38, the existence of many low degree equations [17, first fall degree computations [22 24] and complexity results on the MinRank problem [11. In this paper, we generalize some of these results to polynomial systems arising from a Weil descent.

Experimental Observations. We start our analysis of these systems with an experimental study of their degree of regularity for various parameters $n, m, n^{\prime}, t$. For each set of parameters, we generate a random vector space $V$ of dimension $n^{\prime}$ and a random multivariate polynomial $\mathbf{f}\left(\mathbf{x}_{\mathbf{1}}, \ldots, \mathbf{x}_{\mathbf{m}}\right)$ with degree bounded by $2^{t}-1$ with respect to each variable. We then perform a Weil descent on this

${ }^{1}$ In HFE contexts, the attacker is not given $\mathbf{f}$ but only a "hidden" version of System (2). This can be ignored in the complexity analysis of Gröbner basis algorithms since the hiding transformation only consists of a linear combinations of the equations and a linear change of variables [4838. 
polynomial and we append the field equations to the system. Finally, we apply the Magma function Groebner to the result and we collect the maximal degree $D$ reached during the computation, as given by the Verbose output of the Magma function. We repeat each experiment 100 times.

Table 1. Average maximal degree reached in Gröbner Basis experiments, average computation time (in seconds) and maximal memory requirements (in MB) for random polynomials

\begin{tabular}{|r|r|r|r|r|r|r|r|}
\hline$t$ & $n$ & $n^{\prime}$ & $m$ & $m t+1$ & $D_{a v}$ & Time & Mem. \\
\hline 1 & 6 & 3 & 2 & 3 & 3.1 & 0 & 10 \\
1 & 6 & 2 & 3 & 4 & 3.8 & 0 & 10 \\
1 & 8 & 4 & 2 & 3 & 3.0 & 0 & 11 \\
1 & 12 & 6 & 2 & 3 & 3.6 & 0 & 11 \\
1 & 12 & 4 & 3 & 4 & 4.2 & 0 & 11 \\
1 & 12 & 3 & 4 & 5 & 5.3 & 0 & 14 \\
1 & 12 & 2 & 6 & 7 & 7.4 & 1 & 23 \\
1 & 15 & 5 & 3 & 4 & 4.1 & 5 & 20 \\
1 & 15 & 3 & 5 & 6 & 6.3 & 7 & 114 \\
1 & 16 & 8 & 2 & 3 & 3.0 & 14 & 25 \\
1 & 16 & 4 & 4 & 5 & 5.3 & 16 & 98 \\
1 & 16 & 2 & 8 & 9 & 9.6 & 69 & 3388 \\
1 & 18 & 9 & 2 & 3 & 3.0 & 85 & 74 \\
1 & 18 & 6 & 3 & 4 & 4.1 & 86 & 89 \\
1 & 18 & 3 & 6 & 7 & 7.4 & 233 & 5398 \\
1 & 20 & 10 & 2 & 3 & 3.0 & 487 & 291 \\
1 & 20 & 5 & 4 & 5 & 6.2 & 515 & 733 \\
1 & 20 & 4 & 5 & 6 & 6.2 & 669 & 3226 \\
\hline
\end{tabular}

\begin{tabular}{|r|r|r|r|r|r|r|r|}
\hline$t$ & $n$ & $n^{\prime}$ & $m$ & $m t+1$ & $D_{a v}$ & Time & Mem. \\
\hline 2 & 6 & 3 & 2 & 5 & 5.1 & 0 & 10 \\
2 & 6 & 2 & 3 & 7 & 6.7 & 0 & 10 \\
2 & 8 & 4 & 2 & 5 & 5.1 & 0 & 11 \\
2 & 9 & 3 & 3 & 7 & 7.2 & 0 & 12 \\
2 & 12 & 4 & 3 & 7 & 7.1 & 1 & 38 \\
2 & 12 & 3 & 4 & 9 & 9.3 & 2 & 95 \\
2 & 15 & 5 & 3 & 7 & 7.0 & 12 & 263 \\
2 & 16 & 8 & 2 & 5 & 5.1 & 13 & 36 \\
3 & 6 & 3 & 2 & 7 & 6.6 & 0 & 10 \\
3 & 12 & 6 & 2 & 7 & 7.0 & 1 & 31 \\
3 & 12 & 4 & 3 & 10 & 10.1 & 9 & 70 \\
3 & 12 & 3 & 4 & 13 & 12.6 & 70 & 113 \\
3 & 15 & 5 & 3 & 10 & 10.0 & 118 & 2371 \\
3 & 16 & 8 & 2 & 7 & 7.0 & 23 & 253 \\
3 & 16 & 4 & 4 & 13 & 13.2 & 1891 & 20135 \\
4 & 8 & 4 & 2 & 9 & 8.7 & 1 & 11 \\
4 & 12 & 4 & 3 & 13 & 12.6 & 199 & 116 \\
4 & 15 & 5 & 3 & 13 & 13.1 & 2904 & 6696 \\
\hline
\end{tabular}

Table 1 reports the average value of $D$ for these experiments, as well as the average computation time and the maximal memory used (all experiments were done on an Intel Xeon CPU X5500 processor running at $2.67 \mathrm{GHz}$, with $24 \mathrm{~GB}$ RAM). As is often the case in Gröbner basis computations, our experiments were limited more by the memory requirements than by the computation time.

For all parameter sets, the maximal degrees occuring during Gröbner basis computations were much smaller than the degrees of regularity of regular or semi-regular systems with the same degrees. In fact, our experiments suggest that the degree of regularity of System (2) is not much higher than the value $m t+1$. In other words since the original equations have degree $m t$, the degree of regularity is essentially as small as it could be. The even lower values obtained for all parameter sets such that $t=n^{\prime}$ can be explained by a probable degeneracy in the degrees of the equations. Taking $m=1$, we recover known experimental results on HFE [30].

Heuristic Upper Bound on $\boldsymbol{D}_{\boldsymbol{r e g}}$. As a first step towards explaining these experimental results, we follow Granboulan et al. 38] and we bound of the degree 
of regularity of System (2) from above by the degree of regularity of a smaller system with a lower number of variables. We now suppose that $\left\{\theta_{1}, \ldots, \theta_{n}\right\}$ is a normal basis of $\mathbb{F}_{2^{n}}$ over $\mathbb{F}_{2}$, such that $\theta_{i}:=\theta^{2^{i-1}}$ for some $\theta \in \mathbb{F}_{2^{n}}$. Let $v_{i j} \in \mathbb{F}_{2}$ such that $\mathbf{v}_{\mathbf{i}}=\sum_{j=1}^{n} v_{i j} \theta_{j}$. We define $n m$ auxiliary binary variables $y_{i j}$ such that $\mathbf{x}_{\mathbf{i}}=\sum_{j=1}^{n} y_{i j} \theta_{j}$. Proceeding to a Weil descent as above, we obtain a new system 2

$$
[\mathbf{f}]_{1}^{\downarrow_{y}}=0, \ldots,[\mathbf{f}]_{n}^{\downarrow_{y}}=0
$$

in the variables $y_{i j}$, to which we add $m\left(n+n^{\prime}\right)$ field equations $y_{i j}^{2}-y_{i j}=0$ and $x_{i j}^{2}-x_{i j}=0$, as well as $m n$ linear equations $y_{i j}=\sum_{k=1}^{n} x_{i k} v_{k j}$ modeling the linear constraints. The resulting system of $m\left(n+n^{\prime}\right)$ variables and $n+m(n+$ $\left.n^{\prime}\right)+m n$ equations is equivalent to System (2) (with the field equations), hence they have the same degree of regularity.

Following Granboulan et al. 38, we perform additional modifications on this system to obtain a new system with less variables and higher or equal degree of regularity. First, we observe that linear equations do not contribute to the degree of regularity and can therefore be removed without affecting it. The resulting system is composed of $n+m n$ equations containing only the variables $y_{i j}$ and $m n^{\prime}$ field equations $x_{i j}^{2}-x_{i j}=0$. Without decreasing the degree of regularity, we can focus on the first part containing Equations (3) and the field equations $y_{i j}^{2}-y_{i j}=0$.

In the next step, we observe that the degree of regularity of this system is not affected if we see the variables $y_{i j}$ over $\mathbb{F}_{2^{n}}$ rather than over $\mathbb{F}_{2}$. Thanks to the field equations, the set of solutions is not affected by this change either. We then apply an invertible linear transformation on Equations (3), defined by $F_{i}:=\sum_{j=1}^{n} \theta^{2^{i+j}}[\mathbf{f}]_{j}^{\downarrow_{y}}$ for $i=1, \cdots, n$. This transformation implies $F_{i}=F_{1}^{2^{i-1}}$. Finally, we perform a linear change of variables defined by $z_{i j}:=\sum_{k=1}^{n} \theta^{2^{j+k-1}} y_{i k}$ for $i=1, \ldots, m$, and $j=1, \cdots, n$. Since this corresponds to setting $z_{i 1}=\mathbf{x}_{\mathbf{i}}$, $z_{i 2}=\mathbf{x}_{\mathbf{i}}{ }^{2}, \ldots, z_{i, n}=\mathbf{x}_{\mathbf{i}}{ }^{2-1}$, each $F_{k}$ only depends (linearly) on $z_{i j}, k \leq j \leq$ $t+k-1$. A last linear transformation changes the field equations into $z_{i j}^{2}=z_{i, j+1}$ and $z_{i, n}^{2}=z_{i, 1}$.

Since $F_{2}=F_{1} \cdot F_{1}$ modulo the field equations, the polynomial $F_{2}$ can be expressed at the degree $2 m t$ as an algebraic combination of $F_{1}$ and the field equations. Similarly, all polynomials $F_{i}, i \geq 2$ can be recovered at degree $2 m t$ from algebraic combination of $F_{1}$ and the field equations. Therefore, the degree of regularity of the original system is smaller than the maximum of $2 \mathrm{mt}$ and the degree of regularity of the system $\left\{F_{1}=0 ; z_{i j}^{2}=z_{i, j+1}, i=1, \ldots, m, j=\right.$ $\left.1, \ldots, n-1 ; z_{i, n}^{2}=z_{i, 1}, i=1, \ldots, m\right\}$. Finally like [38, we bound this last degree by the degree of regularity of the subsystem $\left\{F_{1}=0 ; z_{i j}^{2}=z_{i, j+1}, i=\right.$ $1, \ldots, m, j=1, \ldots, t-1\}$. Assuming that this system behaves like a generic system with the same degrees and the same number of variables 3 , its degree of

\footnotetext{
${ }^{2}$ We add a subscript $y$ to the arrows in System (3) to stress that the Weil descent is done on the $y_{i j}$ variables and to distinguish this system from System (2).

${ }^{3}$ A similar assumption of semi-regularity is needed in 38 to apply Bardet's theorem.
} 
regularity can be bounded by $m(2 t-1)$ using Macaulay's bound. Under this heuristic assumption, we conclude that the degree of regularity of System (3) is bounded by $2 m t$.

We point out that the value $2 m t$ is already much below the degree of regularity of a generic system of equations (or even a generic binary system of equations) with the same degrees [6]. Still, our experiments suggest that this bound is not even tight. A tighter bound can be obtained with a seemingly stronger (yet "classical") heuristic assumption.

First Fall Degree. An important characteristic of HFE systems is the existence of many algebraic combinations of the equations that have a degree lower than it would be expected for a generic system. Similar low degree equations were identified for System (2). More precisely, Faugère et al. 3334 showed that for any monomial $\mathbf{m} \in \mathbb{F}_{2^{n}}\left[\mathbf{x}_{\mathbf{1}}, \ldots, \mathbf{x}_{\mathbf{n}^{\prime}}\right]$, the equations obtained by applying a Weil descent on the polynomial $\mathbf{m f}$ are algebraic combinations of the equations of System (2) that produce a degree fall. By the way they are constructed, the existence of these equations is very specific to polynomial systems arising from a Weil descent. For $\mathbf{m}:=\mathbf{x}_{\mathbf{1}}$, we immediately deduce:

Proposition 1. The first fall degree of System (2) is at most $m t+1$.

This proposition provides a heuristic explanation for the degrees of regularity observed above since the first fall degree is often a good approximation of the degree of regularity. As recalled in Section 3, this heuristic assumption is "classical" in algebraic cryptanalysis, and it has in particular been verified for various HFE-like systems 382422 .

Assumption 1. Let $n, m, t, n^{\prime} \in \mathbb{Z}$. Let $\mathbf{f}$ be generated as in our experiments. For all but a negligible fraction of the resulting systems, we have $D_{\text {reg }}=$ $D_{\text {firstfall }}+o\left(D_{\text {firstfall }}\right)$.

The assumption intuitively makes sense for System (2) since not only one but many degree falls are occuring at degree $D_{\text {firstfall }}$ and the next ones (each monomial $\mathbf{m}$ leads to new degree falls).

Heuristic Complexity Bounds for Problem (11). Given the degree of regularity, the complexity of Problem 1 simply follows from the cost of linear algebra.

Proposition 2. If Assumption 1 holds, Problem 1 can be solved with standard Gröbner basis algorithms (like F4 or F5) in time $O\left(n^{\omega D}\right)$ and memory $O\left(n^{2 D}\right)$, where $\omega$ is the linear algebra constant and $D \approx m t$.

In the univariate case, this estimation reduces to $D \approx t$ which perfectly matches known cryptanalysis results on HFE algebraic systems 30 38. Interestingly, the special shape of HFE polynomials (they deploy to quadratic equations over $\mathbb{F}_{2}$ ) seems to have no impact on the degree of regularity (although further restrictions on the shape may have an impact as pointed out in [22]). In the multilinear case, 
the estimation provided by Proposition 2 becomes $D \approx m$ which matches the experimental data of 33 .

As observed in [33.34, the block structure of System (2) can be exploited to accelerate its resolution.

Proposition 3. If Assumption 1 holds, Problem 1 can be solved with block Gröbner basis algorithms in time $O\left(\left(n^{\prime}\right)^{\omega D}\right)$ and memory $O\left(\left(n^{\prime}\right)^{2 D}\right)$, where $\omega$ is the linear algebra constant and $D \approx m t$.

Additional heuristic methods like hybrid approaches (consisting in mixing exhaustive search and polynomial system resolution [529]) may lead to substantial complexity improvements in practice, as was described in 33 . for the multilinear case.

\section{Index Calculus for Elliptic Curves}

We now turn to the main application (so far) of Problem (1). As pointed out in 34, an instance of Problem (11) appears in the relation search step of an index calculus algorithm for elliptic curves proposed by Diem 21. Given a cyclic (additive) group $G$, a generator $P$ of this group and another element $Q$ of $G$, the discrete logarithm problem asks for computing an integer $k$ such that $Q=k P$. Groups typically used in cryptography include the multiplicative groups of finite fields, groups of points on elliptic curves and hyperelliptic curves and Jacobians of higher genus curves. Index calculus algorithms [425] with subexponential complexities have long been obtained for the multiplicative groups of finite fields [1 16 2 5 [39] and more recently for the Jacobian groups of hyperelliptic curves 336 35.

In 2004, Semaev introduced his summation polynomials and identified their potential application to build index calculus algorithms on elliptic curves [51] over prime fields $\mathbb{F}_{p}$. These ideas were independently extended by Gaudry [37] and Diem [20] to elliptic curves over composite fields $\mathbb{F}_{p^{n}}$. Following this approach, Gaudry [37] and later Joux and Vitse [40 41] obtained index calculus algorithms running faster than generic algorithms for any $p$ and any $n \geq 3$. On the other hand, Diem [2021] identified some families of curves with a subexponential time index calculus algorithm by letting $p$ and $n$ grow simultaneously in an appropriate way. As far as was known at the moment, the two families of elliptic curves recommended by standards [4] (elliptic curves over prime fields $\mathbb{F}_{p}$ or over binary fields $\mathbb{F}_{2^{n}}$ with $n$ prime) remained immune to these attacks. In 2012, Faugère et al. 34 observed that the computation of the relations in an algorithm of Diem for binary fields [21] could be reduced to special instances of Problem (1).

Diem's Variant of Index Calculus. Let $K$ be a finite field and let $E$ be an elliptic curve over $K$ defined by the equation $E: y^{2}+x y=x^{3}+\mathbf{a}_{2} x^{2}+\mathbf{a}_{\mathbf{6}}$ for some $\mathbf{a}_{\mathbf{2}}, \mathbf{a}_{\mathbf{6}} \in \mathbb{F}_{2^{n}}$. Semaev's summation polynomials $\mathbf{S}_{\mathbf{r}}$ are multivariate polynomials satisfying $\mathbf{S}_{\mathbf{r}}\left(\mathbf{x}_{\mathbf{1}}, \ldots, \mathbf{x}_{\mathbf{r}}\right)=\mathbf{0}$ for some $\mathbf{x}_{\mathbf{1}}, \ldots, \mathbf{x}_{\mathbf{r}} \in \bar{K}$ if and only 
if there exist $\mathbf{y}_{\mathbf{1}}, \ldots, \mathbf{y}_{\mathbf{r}} \in \bar{K}$ such that $\left(\mathbf{x}_{\mathbf{i}}, \mathbf{y}_{\mathbf{i}}\right) \in E(\bar{K})$ and $\left(\mathbf{x}_{\mathbf{1}}, \mathbf{y}_{\mathbf{1}}\right)+\cdots+$ $\left(\mathbf{x}_{\mathbf{r}}, \mathbf{y}_{\mathbf{r}}\right)=P_{\infty}$ [51]. The summation polynomials can be recursively computed as $\mathbf{S}_{\mathbf{2}}\left(\mathbf{x}_{\mathbf{1}}, \mathbf{x}_{\mathbf{2}}\right):=\mathbf{x}_{\mathbf{2}}+\mathbf{x}_{\mathbf{1}}, \mathbf{S}_{\mathbf{3}}\left(\mathbf{x}_{\mathbf{1}}, \mathbf{x}_{\mathbf{2}}, \mathbf{x}_{\mathbf{3}}\right):=\mathbf{x}_{\mathbf{1}}{ }^{2} \mathbf{x}_{\mathbf{2}}{ }^{2}+\mathbf{x}_{\mathbf{1}}{ }^{2} \mathbf{x}_{\mathbf{3}}{ }^{2}+\mathbf{x}_{\mathbf{1}} \mathbf{x}_{\mathbf{2}} \mathbf{x}_{\mathbf{3}}+$ $\mathbf{x}_{\mathbf{2}}{ }^{2} \mathbf{x}_{\mathbf{3}}{ }^{2}+\mathbf{a}_{\mathbf{6}}$ and for any $r \geq 4$, any $k, 1 \leq k \leq r-3, \quad \mathbf{S}_{\mathbf{r}}\left(\mathbf{x}_{1}, \ldots, \mathbf{x}_{\mathbf{r}}\right):=$ $\operatorname{Res}_{\mathbf{X}}\left(\mathbf{S}_{\mathbf{r}-\mathbf{k}}\left(\mathbf{x}_{\mathbf{1}}, \ldots, \mathbf{x}_{\mathbf{m}-\mathbf{k}-\mathbf{1}}, \mathbf{X}\right), \mathbf{S}_{\mathbf{k}+\mathbf{2}}\left(\mathbf{x}_{\mathbf{r}-\mathbf{k}}, \ldots, \mathbf{x}_{\mathbf{r}}, \mathbf{X}\right)\right)$. For $r \geq 2$, the polynomial $\mathbf{S}_{\mathbf{r}}$ is symmetric and has degree $2^{r-2}$ in every variable $\mathbf{x}_{\mathbf{i}}[51$.

Summation polynomials were used by Gaudry [37], Joux and Vitse [40] and Diem [2021] to compute relations in index calculus algorithms for elliptic curves over composite fields. The following variant is an adaptation of Diem [21].

1. Factor Basis definition. Fix two integers $m, n^{\prime}<n$ with $m n^{\prime} \approx n$ and a vector space $V \subset \mathbb{F}_{2^{n}} / \mathbb{F}_{2}$ of dimension $n^{\prime}$. Let $\mathcal{F}_{V}:=\{(\mathbf{x}, \mathbf{y}) \in E(K) \mid \mathbf{x} \in V\}$ be the factor basis.

2. Relation search. Find about $2^{n^{\prime}}$ relations $a_{i} P+b_{i} Q=\sum_{j=1}^{m} P_{i j}$ with $P_{i j} \in \mathcal{F}_{V}$. For each relation,

(a) Compute $R_{i}:=a_{i} P+b_{i} Q$ for random integers $a_{i}, b_{i}$.

(b) Solve Semaev's polynomial $\mathbf{S}_{\mathbf{m}+\mathbf{1}}\left(\mathbf{x}_{\mathbf{1}}, \ldots, \mathbf{x}_{\mathbf{m}},\left(R_{i}\right)_{x}\right)$ with the constraints $\mathbf{x}_{\mathbf{i}} \in V$.

(c) If there is no solution, go back to (a).

3. Linear Algebra. Perform linear algebra on the relations to recover the discrete logarithm value.

In previous works [37202140, a Weil descent was applied to Semaev's polynomials and the resulting systems were solved with resultants or Gröbner basis algorithms. In these works, the complexity of the relation search step was derived from the complexity of solving generic systems. However as pointed out in 33.34 and further demonstrated in Section 4 of the present paper, polynomial systems arising from a Weil descent are very far from generic ones.

A New Complexity Analysis. We now revisit Diem's algorithm [21] and its analysis by 34 in accordance with our new analysis of Problem (1). Let $n, m, n^{\prime}$ be integer numbers. Before starting Diem's algorithm, the $(m+1)$ th summation polynomial must be computed. Using Collins' evaluation/interpolation method [15] for the resultant, this can be done in time approximately $2^{t_{1}}$ where 4 $t_{1} \approx m(m+1)$. We then compute about $2^{n^{\prime}}$ relations. To obtain these relations, we solve special instances of Problem (1) where $\mathbf{f}\left(\mathbf{x}_{\mathbf{1}}, \ldots, \mathbf{x}_{\mathbf{m}}\right):=\mathbf{S}_{\mathbf{m}+\mathbf{1}}\left(\mathbf{x}_{\mathbf{1}}, \ldots\right.$, $\left.\mathbf{x}_{\mathbf{m}},\left(a_{i} P+b_{i} Q\right)_{x}\right)$ has degree $2^{m-1}$ with respect to every variable. Since Semaev's polynomials are clearly not random ones, we perform additional experiments.

In our experiments, we apply Diem's algorithm to a randomly chosen binary curve $E: y^{2}+x y=x^{3}+\mathbf{a}_{2} x^{2}+\mathbf{a}_{\mathbf{6}}$ defined over $\mathbb{F}_{2^{n}}$, where $n \in\{11,17\}$. We first fix $m \in\{2,3\}$ and $n^{\prime}:=\lceil n / m\rceil$. We then generate a random vector space $V$ of dimension $n^{\prime}$ and a random point $R$ on the curve such that $\mathbf{f}$ has solutions. As in Section 4, we finally use the Groebner function of Magma to solve Semaev's

\footnotetext{
${ }^{4}$ To compute $\mathbf{S}_{\mathbf{m}+\mathbf{1}}$, we apply Collins' algorithm on $S_{k}$ where $k=\left\lceil\frac{m+3}{2}\right\rceil$. This polynomial has degree $2^{\lceil(m-1) / 2\rceil}$ in each variable. Following Collins, Theorem 9, we have $t_{1} \leq 2(m+1) m / 2=m(m+1)$.
} 
Table 2. Average maximal degree reached in Gröbner Basis experiments, average computation time (in seconds) and maximal memory requirements (in MB) for Semaev polynomials. (R): Random curves. (K): Koblitz curves.

\begin{tabular}{|c|c|c|c|c|c|c|c|c|}
\hline$E$ & $n$ & $n^{\prime}$ & $m$ & $t$ & $m t+1$ & $D_{a v}$ & Time & Mem. \\
\hline $\mathrm{K}$ & 11 & 6 & 2 & 2 & 5 & 3.0 & 0 & 11 \\
$\mathrm{~K}$ & 11 & 4 & 3 & 3 & 10 & 7.1 & 1 & 15 \\
$\mathrm{~K}$ & 17 & 9 & 2 & 2 & 5 & 4.0 & 0 & 15 \\
$\mathrm{~K}$ & 17 & 6 & 3 & 3 & 10 & 7.2 & 132 & 2133 \\
\hline
\end{tabular}

\begin{tabular}{|c|c|c|c|c|c|c|c|c|}
\hline$E$ & $n$ & $n^{\prime}$ & $m$ & $t$ & $m t+1$ & $D_{a v}$ & Time & Mem. \\
\hline $\mathrm{R}$ & 11 & 6 & 2 & 2 & 5 & 3.0 & 0 & 11 \\
$\mathrm{R}$ & 11 & 4 & 3 & 3 & 10 & 7.1 & 1 & 15 \\
$\mathrm{R}$ & 17 & 9 & 2 & 2 & 5 & 4.0 & 0 & 16 \\
$\mathrm{R}$ & 17 & 6 & 3 & 3 & 10 & 7.1 & 130 & 2136 \\
\hline
\end{tabular}

equation $\mathbf{S}_{\mathbf{m}+\mathbf{1}}\left(\mathbf{x}_{\mathbf{1}}, \ldots, \mathbf{x}_{\mathbf{m}}, R_{x}\right)=\mathbf{0}$ with the linear constraints. We repeat this experiment 100 times for each parameter set, then we repeat all our experiments with the Koblitz curve $E: y^{2}+x y=x^{3}+x^{2}+1$. The average value of the maximal degrees reached during the computation, the average computation time and the maximal memory requirements are reported in Table 2 .

In all cases, the maximal degrees reached in the computations were even below the first fall degree bound given by Proposition (11). This phenomenon is probably due to the sparsity of Semaev's polynomials and will be exploited in future work (in particular, the degree of $\mathbf{S}_{\mathbf{m}+\mathbf{1}}$ with respect to every variable is $2^{m-1}$ but bounded by $2^{m}-1$ in the analysis of Section 4). From now on in the analysis, we ignore this difference and analyze Semaev's polynomials as the random polynomials of Section 4 .

Assumption 2. Assumption 11 still holds if $\mathbf{f}$ is generated from Semaev's polynomials as in the experiments of this section.

Under Assumption (2), Step 2(b) of Diem's algorithm can be solved using a dedicated Gröbner basis algorithm taking advantage of the block structure, in a time $\left(n^{\prime}\right)^{\omega D}$, where $D \approx\left(m^{2}+1\right)$ and $\omega$ is the linear algebra constant. Once the $x$ components of a relation have been computed, the $y$ components can be found by solving $m$ quadratic equations and testing each possible combination of the solutions. This requires a time roughly $2^{m}$, that can be neglected. On average, the probability that a point $R_{i}:=a_{i} P+b_{i} Q$ can be written as a sum of $m$ points from the factor basis can be heuristically approximated by $\frac{2^{m n^{\prime}-n}}{m !}[21$. Assuming $m n^{\prime} \approx n$, the total cost of the relation search step can therefore be approximated by $2^{t_{2}}$, where $t_{2} \approx m \log m+n^{\prime}+\omega\left(m^{2}+1\right) \log n^{\prime}$.

The last step of Diem's algorithm consists in (sparse) linear algebra on a matrix of rank about $2^{n^{\prime}}$ with about $m$ elements of size about $n$ bits per row. This step takes a time approximately equal to $m n 2^{\omega^{\prime} n^{\prime}}=2^{t_{3}}$, where $t_{3} \approx \log m+$ $\log n+\omega^{\prime} n^{\prime}$ and $\omega^{\prime}$ is the sparse linear algebra constant. If Assumption (2) holds and if $m n^{\prime} \approx n$, the total time taken by Diem's algorithm can be estimated by $T:=2^{t_{1}}+2^{t_{2}}+2^{t_{3}}$, where $t_{1}, t_{2}, t_{3}$ are defined as above.

On the Hardness of ECDLP in Characteristic 2. We now evaluate the hardness of the elliptic curve discrete logarithm problem over the field $\mathbb{F}_{2^{n}}$ for "small" values of $n$. In our estimations, we use $\omega=\log (7) / \log (2)$ and $\omega^{\prime}=2$. 
Table 3. Complexity estimates for Diem's algorithm in characteristic 2

\begin{tabular}{|c|c|c|c|c|c|c|}
\hline$n$ & $m$ & $n^{\prime}$ & $t_{1}$ & $t_{2}$ & $t_{3}$ & $t_{\max }$ \\
\hline 50 & 2 & 25 & 6 & 92 & 57 & 92 \\
100 & 2 & 50 & 6 & 131 & 108 & 131 \\
160 & 2 & 80 & 6 & 171 & 168 & 171 \\
200 & 2 & 100 & 6 & 195 & 209 & 209 \\
500 & 3 & 167 & 12 & 379 & 344 & 379 \\
1000 & 4 & 250 & 20 & 638 & 512 & 638 \\
\hline
\end{tabular}

\begin{tabular}{|c|c|c|c|c|c|c|}
\hline$n$ & $m$ & $n^{\prime}$ & $t_{1}$ & $t_{2}$ & $t_{3}$ & $t_{\max }$ \\
\hline 2000 & 4 & 500 & 20 & 936 & 1013 & 1013 \\
2500 & 5 & 500 & 30 & 1166 & 1014 & 1166 \\
5000 & 6 & 833 & 42 & 1857 & 1682 & 1857 \\
10000 & 7 & 1429 & 56 & 2919 & 2873 & 2919 \\
20000 & 9 & 2222 & 90 & 4810 & 4462 & 4810 \\
50000 & 12 & 4167 & 156 & 9105 & 8353 & 9105 \\
\hline
\end{tabular}

We consider $n \in\left\{50,100,160,200,500,1000,2000,2500,5000,10^{4}, 2 \cdot 10^{4}, 5\right.$. $\left.10^{4}, 10^{5}, 2 \cdot 10^{5}, 5 \cdot 10^{5}, 10^{6}\right\}$ and $m \in\{2, \ldots, n / 2\}$. For every pair of values, we compute values $t_{1}, t_{2}$ and $t_{3}$ as above. Finally, we approximate the total running time of Diem's algorithm by $2^{t_{\max }}$ where $t_{\max }:=\max \left(t_{1}, t_{2}, t_{3}\right)$. For every value of $n$, Table 3 presents the data corresponding to the value $m$ for which $t_{\max }$ is minimal. We point out that the numbers obtained here have to be taken cautiously since they all rely on Assumption 2 and involve some approximations.

According to our estimations, Diem's version of index calculus (together with a sparse Gröbner basis algorithm) beats generic algorithms for any $n \geq N$, where $N$ is an integer close to 2000. An actual attack for current cryptographically recommended parameters $(n \approx 160)$ seems to be out of reach today, but the numbers in 34 suggest that medium-size parameters could be reachable with additional Gröbner basis heuristics like the hybrid method 9]. Large prime variations 35] of Diem's algorithm may also lead to substantial improvements in practice. This will be investigated in further work.

Letting $n$ grow and fixing $n^{\prime}:=n^{\alpha}$ and $m:=n^{1-\alpha}$ for a positive constant $\alpha<1$, we obtain

$$
\begin{aligned}
& t_{1} \approx n^{2(1-\alpha)} \\
& t_{2} \approx(1-\alpha) n^{1-\alpha} \log n+n^{\alpha}+\alpha \omega n^{2(1-\alpha)} \log n \\
& t_{3} \approx(2-\alpha) \log n+\omega^{\prime} n^{\alpha}
\end{aligned}
$$

Taking $\alpha:=2 / 3$, the relation search dominates the complexity of the index calculus algorithm and we deduce the following result 5

Proposition 4. Under Assumption 2, the discrete logarithm problem over $\mathbb{F}_{2^{n}}$ can asymptotically be solved in time $O\left(2^{c n^{2 / 3} \log n}\right)$, where $c:=2 \omega / 3$ and $\omega$ is the linear algebra constant.

In particular if the Gaussian elimination algorithm is used for linear algebra, we have $\omega=3$ and $c=2$. We stress that Proposition 4 holds even when $n$ is prime. Until now, the best complexity estimates obtained in that case corresponded to generic algorithms that run in time $2^{n / 2}$.

\footnotetext{
${ }^{5}$ Note that the weaker bound $D_{\text {reg }} \leq 2 m t$ derived in Section 4 with Macaulay's bound also leads to a subexponential complexity but with a constant $c=4 \omega / 3$.
} 


\section{Conclusion and Perspectives}

In this paper, we revisited the complexity of solving polynomial systems arising from a Weil descent, a class of polynomial systems previously introduced by Faugère et al. 33 34. We observed that these systems can be seen as natural extensions of HFE systems and we generalized various results on HFE. Based on experimental results and heuristic arguments, we conjectured that the degree of regularity of these systems are only slightly larger than their original degrees, and we deduced new heuristic bounds on their resolution. Interestingly, our bounds nicely generalize previous bounds on HFE.

The most proeminent consequence of our analysis so far concerns the elliptic curve discrete logarithm problem (ECDLP) over binary fields. Indeed, our heuristic analysis suggests that ECDLP can be solved in subexponential time $O\left(2^{c n^{2 / 3} \log n}\right)$ over the binary field $\mathbb{F}_{2^{n}}$, where $c$ is a constant smaller than 2 . This complexity is obtained with an index calculus algorithm due to Diem [20] and a block-structured Gröbner basis algorithm. In practice, our estimations predict that the resulting algorithm is faster than generic algorithms (previously thought to be the best algorithms for this problem) for any $n$ larger than $N$, where $N$ is an integer approximately equal to 2000. In particular, binary elliptic curves of currently recommended sizes $(n \approx 160)$ are not immediately threatened.

Our complexity estimates are based on heuristic assumptions that differ from other index calculus algorithms, but are common in algebraic cryptanalysis. The polynomial systems appearing in the cryptanalysis of HFE have been intensively studied in the last 15 years, yet we have no definitive proof for their commonly admitted complexity. Our paper broadens the interest of these researches to all polynomial systems arising from a Weil descent and to their various applications. We leave further experimental and theoretical investigation of our heuristic assumptions to further work.

To conclude this paper, we point out that most of our results generalize quite easily to other fields, resulting in comparable asymptotic complexities.

Acknowledgements. We are indebted to Sylvie Baudine and the anonymous reviewers for their help in improving this paper. We also thank Jean-Charles Faugère, Ludovic Perret and Guénaël Renault for their useful comments on a preliminary version of this paper. Finally, Christophe Petit would like to thank Daniel Augot for his hospitality since this paper was partially written at LIX.

\section{References}

1. Leonard, M.A.: A subexponential algorithm for the discrete logarithm problem with applications to cryptography (abstract). In: FOCS, pp. 55-60. IEEE (1979)

2. Leonard, M.A.: The function field sieve. In: Adleman, Huang [4], pp. 108-121

3. Leonard, M.A., DeMarrais, J., Huang, M.-D.A.: A subexponential algorithm for discrete logarithms over the rational subgroup of the Jacobians of large genus hyperelliptic curves over finite fields. In: Adleman, Huang, [4] pp. 28-40 
4. Huang, M.-D.A., Adleman, L.M. (eds.): ANTS 1994. LNCS, vol. 877. Springer, Heidelberg (1994)

5. Adleman, L.M., Huang, M.-D.A.: Function field sieve method for discrete logarithms over finite fields. Inf. Comput. 151(1-2), 5-16 (1999)

6. Bardet, M.: Etude des systèmes algébriques surdéterminés. Applications aux codes correcteurs et à la cryptographie. PhD thesis, Université Paris 6 (2004)

7. Bardet, M., Faugère, J.-C., Salvy, B.: On the complexity of Gröbner basis computation of semi-regular overdetermined algebraic equations. In: International Conference on Polynomial System Solving, ICPSS, pp. 71-75 (November 2004)

8. Bardet, M., Faugère, J.-C., Salvy, B.: Asymptotic expansion of the degree of regularity for semi-regular systems of equations. In: Gianni, P. (ed.) The Effective Methods in Algebraic Geometry Conference, Mega 2005, pp. 1-14 (May 2005)

9. Bettale, L., Faugère, J.-C., Perret, L.: Hybrid approach for solving multivariate systems over finite fields. Journal of Mathematical Cryptology 3(3), 177-197 (2010)

10. Bettale, L., Faugère, J.-C., Perret, L.: Cryptanalysis of multivariate and oddcharacteristic hfe variants. In: Catalano, et al. [14], pp. 441-458

11. Bettale, L., Faugère, J.-C., Perret, L.: Cryptanalysis of HFE, Multi-HFE and Variants for Odd and Even Characteristic. Des. Codes Cryptography, 1-42 (accepted, 2012)

12. Bouillaguet, C., Faugère, J.-C., Fouque, P.-A., Perret, L.: Practical cryptanalysis of the identification scheme based on the isomorphism of polynomial with one secret problem. In: Catalano, et al. [14], pp. 473-493

13. Buchberger, B.: Ein Algorithmus zum Auffinden der Basiselemente des Restklassenringes nach einem nulldimensionalen Polynomideal. PhD thesis, Universität Innsbruck (1965)

14. Catalano, D., Fazio, N., Gennaro, R., Nicolosi, A. (eds.): PKC 2011. LNCS, vol. 6571, pp. 2011-2014. Springer, Heidelberg (2011)

15. Collins, G.: The calculation of multivariate polynomial resultants. Journal of the Association for Computing Machinery 18, 515-522 (1971)

16. Coppersmith, D.: Fast evaluation of logarithms in fields of characteristic two. IEEE Transactions on Information Theory 30(4), 587-593 (1984)

17. Courtois, N.T.: The Security of Hidden Field Equations (HFE). In: Naccache, D. (ed.) CT-RSA 2001. LNCS, vol. 2020, pp. 266-281. Springer, Heidelberg (2001)

18. Courtois, N., Klimov, A., Patarin, J., Shamir, A.: Efficient Algorithms for Solving Overdefined Systems of Multivariate Polynomial Equations. In: Preneel, B. (ed.) EUROCRYPT 2000. LNCS, vol. 1807, pp. 392-407. Springer, Heidelberg (2000)

19. Cox, D., Little, J., O'Shea, D.: Ideals, Varieties, and Algorithms, 1st edn. Springer, Heidelberg (1992)

20. Diem, C.: On the discrete logarithm problem in elliptic curves. Compositio Mathematica 147, 75-104 (2011)

21. Diem, C.: On the discrete logarithm problem in elliptic curves II (2011), http://www.math.uni-leipzig.de/ diem/preprints/dlp-ell-curves-II.pdf

22. Ding, J., Hodges, T.J.: Inverting HFE Systems Is Quasi-Polynomial for All Fields. In: Rogaway, P. (ed.) CRYPTO 2011. LNCS, vol. 6841, pp. 724-742. Springer, Heidelberg (2011)

23. Ding, J., Kleinjung, T.: Degree of regularity for HFE-. IACR Cryptology ePrint Archiv, 2011:570 (2011)

24. Dubois, V., Gama, N.: The Degree of Regularity of HFE Systems. In: Abe, M. (ed.) ASIACRYPT 2010. LNCS, vol. 6477, pp. 557-576. Springer, Heidelberg (2010)

25. Enge, A., Gaudry, P.: A general framework for subexponential discrete logarithm algorithms. Acta Arith. 102(1), 83-103 (2002) 
26. Faugère, J.-C.: A new efficient algorithm for computing Gröbner bases (F4). Journal of Pure and Applied Algebra 139(1-3), 61-88 (1999)

27. Faugère, J.-C.: A new efficient algorithm for computing Gröbner bases without reduction to zero (F5). In: Proceedings of the 2002 International Symposium on Symbolic and Algebraic Computation, ISSAC 2002, pp. 75-83. ACM, New York (2002)

28. Faugère, J.-C., Din, M.S.E., Spaenlehauer, P.-J.: Computing loci of rank defects of linear matrices using gröbner bases and applications to cryptology. In: ISSAC, pp. 257-264 (2010)

29. Faugère, J.-C., Din, M.S.E., Spaenlehauer, P.-J.: Gröbner bases of bihomogeneous ideals generated by polynomials of bidegree $(1,1)$ : Algorithms and complexity. J. Symb. Comput. 46(4), 406-437 (2011)

30. Faugère, J.-C., Joux, A.: Algebraic Cryptanalysis of Hidden Field Equation (HFE) Cryptosystems Using Gröbner Bases. In: Boneh, D. (ed.) CRYPTO 2003. LNCS, vol. 2729, pp. 44-60. Springer, Heidelberg (2003)

31. Faugère, J.-C., Otmani, A., Perret, L., Tillich, J.-P.: Algebraic Cryptanalysis of McEliece Variants with Compact Keys. In: Gilbert, H. (ed.) EUROCRYPT 2010. LNCS, vol. 6110, pp. 279-298. Springer, Heidelberg (2010)

32. Faugère, J.-C., Perret, L.: Polynomial Equivalence Problems: Algorithmic and Theoretical Aspects. In: Vaudenay, S. (ed.) EUROCRYPT 2006. LNCS, vol. 4004, pp. 30-47. Springer, Heidelberg (2006)

33. Faugère, J.-C., Perret, L., Petit, C., Renault, G.: New subexponential algorithms for factoring in $S L\left(2, \mathbb{F}_{2^{n}}\right)$. Cryptology ePrint Archive, Report 2011/598 (2011), http://eprint.iacr.org/

34. Faugère, J.-C., Perret, L., Petit, C., Renault, G.: Improving the complexity of index calculus algorithms in elliptic curves over binary fields. In: Pointcheval, Johansson [50], pp. 27-44

35. Gaudry, P., Thomé, E., Thériault, N., Diem, C.: A double large prime variation for small genus hyperelliptic index calculus. Math. Comp. 76(257), 475-492 (electronic) (2007)

36. Gaudry, P.: An Algorithm for Solving the Discrete Log Problem on Hyperelliptic Curves. In: Preneel, B. (ed.) EUROCRYPT 2000. LNCS, vol. 1807, pp. 19-34. Springer, Heidelberg (2000)

37. Gaudry, P.: Index calculus for abelian varieties of small dimension and the elliptic curve discrete logarithm problem. J. Symb. Comput. 44(12), 1690-1702 (2009)

38. Granboulan, L., Joux, A., Stern, J.: Inverting HFE Is Quasipolynomial. In: Dwork, C. (ed.) CRYPTO 2006. LNCS, vol. 4117, pp. 345-356. Springer, Heidelberg (2006)

39. Joux, A., Lercier, R.: The Function Field Sieve in the Medium Prime Case. In: Vaudenay, S. (ed.) EUROCRYPT 2006. LNCS, vol. 4004, pp. 254-270. Springer, Heidelberg (2006)

40. Joux, A., Vitse, V.: Elliptic Curve Discrete Logarithm Problem over Small Degree Extension Fields. Application to the static Diffie-Hellman problem on $E\left(\mathbb{F}_{q^{5}}\right)$. Cryptology ePrint Archive, Report 2010/157. Journal of Cryptology (2010), http://eprint.iacr.org/

41. Joux, A., Vitse, V.: Cover and decomposition index calculus on elliptic curves made practical - application to a previously unreachable curve over $\mathbb{F}_{p^{6}}$. In: Pointcheval, Johansson [50], pp. 9-26

42. Kipnis, A., Shamir, A.: Cryptanalysis of the HFE Public Key Cryptosystem by Relinearization. In: Wiener, M. (ed.) CRYPTO 1999. LNCS, vol. 1666, pp. 19-30. Springer, Heidelberg (1999) 
43. Kraitchik, M.: Théorie des nombres. Gauthier-Villars (1922)

44. Lazard, D.: Gröbner-Bases, Gaussian Elimination and Resolution of Systems of Algebraic Equations. In: van Hulzen, J.A. (ed.) ISSAC 1983 and EUROCAL 1983. LNCS, vol. 162, pp. 146-156. Springer, Heidelberg (1983)

45. Macaulay, F.S.: The algebraic theory of modular systems. Cambridge Mathematical Library, vol. XXXI. Cambridge University Press (1916)

46. Macaulay, F.S.: Some properties of enumeration in the theory of modular systems. Proc. London Math. Soc. 26, 531-555 (1927)

47. National Institute of Standards and Technology. Digital Signature Standard (DSS). Federal Information Processing Standards Publication 186-3 (2009)

48. Patarin, J.: Hidden Fields Equations (HFE) and Isomorphisms of Polynomials (IP): Two New Families of Asymmetric Algorithms. In: Maurer, U.M. (ed.) EUROCRYPT 1996. LNCS, vol. 1070, pp. 33-48. Springer, Heidelberg (1996)

49. Petit, C., Quisquater, J.-J.: On polynomial systems arising from a weil descent. Cryptology ePrint Archive, Report 2012/146 (2012), http://eprint.iacr.org/

50. Pointcheval, D., Johansson, T. (eds.): EUROCRYPT 2012. LNCS, vol. 7237, pp. 2012-2031. Springer, Heidelberg (2012)

51. Semaev, I.: Summation polynomials and the discrete logarithm problem on elliptic curves (2004), http://www.isg.rhul.ac.uk/ ppai034/_pub/papers/Semaev\%20\%28Feb\%29.pdf

52. Yang, B.-Y., Chen, J.-M., Courtois, N.T.: On Asymptotic Security Estimates in XL and Gröbner Bases-Related Algebraic Cryptanalysis. In: López, J., Qing, S., Okamoto, E. (eds.) ICICS 2004. LNCS, vol. 3269, pp. 401-413. Springer, Heidelberg (2004) 